\title{
Quality Model for the Evaluation of Geospatial Web Services within the Spatial Data Infrastructure of Uruguay
}

\author{
Martín de los Reyes, Laura González, Raquel Sosa \\ Instituto de Computación, Facultad de Ingeniería \\ Universidad de la República \\ J. H. y Reissig 565, Montevideo, Uruguay \\ tincho1448@gmail.com, \{lauragon, raquels\}@fing.edu.uy
}

\begin{abstract}
Resumen-Quality of Service (QoS), applied in particular to Web Services, is an area of increasing interest. One approach to deal with QoS issues is the identification of quality characteristics, usually documented in quality models. Geospatial Web Services are standardized service interfaces which facilitate the processing and exchange of distributed geographic information. These services represent the technological backbone of Spatial Data Infrastructures (SDI), which are data-exchange frameworks geared towards promoting the availability and exploitation of geographic information. This paper proposes a quality model for the evaluation of Geospatial Web Services within IDEuy: the SDI of Uruguay. The development of this model was based on requirements pertaining to the Uruguayan context, leading international SDI initiatives and related work. The application of the model to evaluate services within IDEuy is also presented.
\end{abstract}

Palabras Clave-Geospatial Web Services, Spatial Data Infrastructure, Quality of Service, Quality Model.

\section{INTRODUCCIÓN}

En la actualidad hay un claro acuerdo para especificar los aspectos funcionales de los Servicios Web. Sin embargo, en los últimos años el interés se ha volcado a los aspectos no funcionales o de calidad de servicio (Quality of Service, QoS) [1]. Un enfoque para abordar este tema es la identificación y clasificación de características de calidad [2][3] (p. ej. disponibilidad), las cuales se organizan generalmente de forma jerárquica y se documentan en modelos de calidad [1].

Por otro lado, los Servicios Web Geográficos son estándares elaborados en su mayoría por el Open Geospatial Consortium ${ }^{1}$ (OGC), como parte de una arquitectura orientada al procesamiento e intercambio de información geográfica distribuida. Estos estándares son elementos fundamentales para las Infraestructuras de Datos Espaciales (IDE) [4], las cuales son plataformas para permitir el uso y publicación de información geográfica a distintas escalas (p. ej. organización, país, región).

En particular, la IDEuy ${ }^{2}$ es la IDE de alcance nacional de Uruguay. Esta IDE consiste en una plataforma con una arquitectura federada compuesta por nodos de diferentes organismos que comparten información geográfica a través de

\footnotetext{
${ }^{1} \mathrm{http}: / /$ www.opengeospatial.org/

${ }^{2}$ http://ide.uy/
}

Servicios Web Geográficos. En este esquema los organismos públicos generadores de la información geográfica la publican para el uso de los demás organismos públicos o particulares.

Si bien existen trabajos que abordan la temática de calidad en el área de servicios web geográficos [5][6][7], no hay propuestas de modelos de calidad para evaluar este tipo de servicios en el contexto de una IDE y, más en particular, en el contexto uruguayo de acuerdo a lo establecido por la IDEuy.

En este artículo se propone un Modelo de Calidad para la Evaluación de Servicios Web Geográficos en la IDEuy.

El modelo se construyó, en primer lugar, identificando características de calidad en tres tipos de fuentes: i) documentación, normativas y referentes del contexto uruguayo, en particular, de la IDEuy y de la Plataforma de Gobierno Electrónico de Uruguay, ii) documentación y normativas de IDEs de referencia, y iii) trabajo relacionado en el área.

En segundo lugar, dichas características se consolidaron en un modelo de calidad organizado en siete dimensiones: Seguridad, Confiabilidad, Rendimiento, Interoperabilidad, Publicación Datos, Metadatos y Usabilidad. Por ejemplo, la dimensión Publicación Datos incluye características como Datos Abiertos y Área de Cobertura. Para cada característica se definió un conjunto de métricas que son los instrumentos utilizados para medir dichas características. Siguiendo con el ejemplo anterior, para Datos Abiertos se definió la métrica Grado de Apertura.

Por último, se aplicó el modelo para evaluar cinco servicios de la IDEuy. Para esto se implementaron métodos de medición para un subconjunto de las métricas, se obtuvieron mediciones de dichas métricas a través de la ejecución de los métodos y se compararon estas mediciones con los valores esperados.

El resto del documento se organiza de la siguiente manera. En la Sección II se presenta el marco conceptual relevante para este trabajo. En la Sección III se identifican características de calidad para los Servicios Web Geográficos de la IDEuy. En la Sección IV se propone el Modelo de Calidad en base a la características identificadas. En la Sección V se presenta la aplicación del modelo para evaluar servicios de la IDEuy. En la Sección VI se analiza trabajo relacionado y, por último, en la Sección VII se presentan conclusiones y trabajo a futuro. 


\section{MARCO CONCEPTUAL}

\section{A. Datos Espaciales}

Se consideran Datos Espaciales [8] a cualquier conjunto de datos que contenga información de referencia geoespacial. Conceptualmente se categorizan estos datos en Vectoriales y datos Raster que tienen forma de grilla o matriz. Los datos Vectoriales corresponden a objetos geométricos (p. ej. puntos, líneas, polígonos) que tienen asociados datos alfanuméricos. En los datos Raster cada celda es un área regular de la superficie terrestre a determinada escala y se representa con un pixel a determinada resolución: un pixel puede respresentar desde centímetros hasta kilómetros cuadrados. En los datos Raster se almacena en general un solo valor asociado a cada celda.

Para georreferenciar los Datos Espaciales se usan Sistemas de Referencias Espaciales (SRS) o Sistemas de Referencias de Coordenadas (CRS) [8]. Estos permiten asociar coordenadas a una ubicación en la tierra siendo el más conocido LatitudLongitud. Hay otros sistemas que son proyecciones planas locales a ciertas zonas de la tierra para lograr mayor exactitud en las medidas de distancias. Para trabajar con diferentes capas de datos se necesita que todas estén en el mismo CRS [9].

Los datos espaciales se pueden almacenar e intercambiar en diferentes formatos: Archivos Shape (i.e. formato para información vectorial definido por ESRI), codificaciones XML (GML[10] o KML[11]), entre otros. Los datos Raster suelen almacenarse e intercambiarse en formatos enriquecidos de imagen (p. ej. GeoTIFF). También se usan formatos livianos como JPG o PNG, para generar vistas (i.e. datos representados según una simbología) [9].

\section{B. Calidad de Servicios Web}

Durante los últimos años el interés de la industria y de la academia se ha volcado a los aspectos no funcionales, también llamados de calidad, de los servicios web [1]. La calidad de servicio (Quality of Service, QoS) cumple un rol muy importante en todo su ciclo de vida dado que se puede utilizar para seleccionar entre servicios funcionalmente equivalentes así como para establecer acuerdos de nivel de servicio (Service Level Agreement, SLA) y monitorear su cumplimiento [1].

La identificación y clasificación de características de calidad (p. ej. tiempo de respuesta) es una de las actividades principales cuando se quieren abordar aspectos de QoS [3]. Estas características se organizan generalmente de forma jerárquica y se documentan en modelos de calidad. La mayoría de las propuestas de modelos de QoS [1][12][2][13] identifican características similares aunque presentan algunas diferencias en su semántica.

La forma en que se organizan los modelos de calidad, por ejemplo los de QoS, varía dependiendo de la propuesta. En particular, en [14] se propone un metamodelo de calidad que define una organización en base a dimensiones, factores, métricas y métodos. Una dimensión captura una faceta de calidad a alto nivel (p. ej. Rendimiento) mientras que un factor representa un aspecto particular de una dimensión (p. ej. Tiempo de Respuesta). Una métrica es un instrumento utilizado para medir un factor de calidad (p. ej. tiempo de respuesta promedio diario) y un método es un proceso que implementa una determinada métrica (p. ej. un programa que invoca a un servicio para conocer su tiempo de respuesta).

\section{Servicios Web Geográficos}

Los Servicios Web Geográficos son estándares de Web Services desarrollados por el Open Geospatial Consortium (OGC). Al consultar datos espaciales a través de Servicios Web Geográficos se tienen interfaces bien definidas, similares a los servicios web REST. Web Map Service (WMS) [15] permite obtener mapas en formato de imagen y realizar consultas puntuales de sus atributos descriptivos. Web Feature Service (WFS) [16] brinda operaciones de consulta más complejas sobre datos vectoriales. Permite definir filtros, recibir información detallada en formatos estándar como GML o KML y también realizar operaciones de altas, bajas y modificaciones sobre los datos en el servidor. Para acceder a datos Raster se usa Web Coverage Service (WCS) [17], y para acceder a Metadatos se usa Catalogue Service for the Web (CSW) [18].

Todos los estándares de Servicios Web OGC tienen un método getCapabilities que da información del servicio, datos disponibles, formatos de datos, sistemas de referencia y otros detalles de configuración específica de cada tipo de servicio.

El estándar WMS se utiliza para producir y transportar mapas generados de forma dinámica a partir de información geográfica. El estándar define como "mapa" a la representación gráfica de información geográfica en formato de archivo digital en una imagen, en formato PNG o JPEG, por ejemplo. Además del GetCapabilities, los métodos de este servicio son:

- GetMap: devuelve una imagen del mapa de acuerdo a los parámetros especificados.

- GetFeatureInfo: devuelve información sobre una entidad en particular.

El estándar WFS define un servicio utilizado para consultar y/o editar entidades geográficas vectoriales. Un servicio WFS básico permite realizar consultas y obtener entidades (features) en formato GML. Un servicio WFS transaccional (WFS-T) permite a los clientes crear, actualizar o eliminar entidades del servidor. Además del GetCapabilities, los principales métodos de este servicio son:

- GetFeature: Devuelve la selección de features de una fuente de datos, incluyendo la geometría y sus atributos.

- DescribeFeatureType: Describe los atributos disponibles para una entidad.

- Transaction: Permite modificar entidades, permitiendo insertar, actualizar o eliminar.

El estándar CSW define un servicio que permite describir, buscar y consultar metadatos relacionados con los datos, servicios y recursos de tipo geográfico. Además del GetCapabilities, los principales métodos de este servicio son:

- GetDomain: Permite a los usuarios consultar sobre el rango de alguna propiedad o parámetro determinado.

- GetRecordsById: devuelve los registros de metadatos que tiene un recurso a través de su identificador. 
- GetRecords: Envía una consulta al catálogo (query) y retorna los datos correspondientes.

\section{Infraestructura de Datos Espaciales}

Una Infraestructura de Datos Espaciales (IDE) [4] es una iniciativa que organiza el acceso a los Datos Espaciales que posee una Organización (o varias). Para definir una IDE se consideran cinco elementos: los Datos, Las Leyes o Reglamentaciones, los Estándares a usar, las Tecnologías a usar y las Partes Interesadas (Organizaciones y Personas). Las IDEs pueden tener diferentes alcances, desde una sola organización, un Departamento, un País o un grupo de Países.

Los principales elementos que componen una IDE son [19]:

- Datos: Los datos deben estar al alcance de los usuarios con las restricciones de uso que decida el propietario. Dentro de los datos de una IDE se pueden encontrar dos tipos de datos: datos de referencia y los datos temáticos. Los datos de referencia son los que forman un mapa base y los datos temáticos son los que proporcionan información sobre un área específica.

- Marco legal: Regula algunos aspectos como qué información es oficial, derechos de autor, licencias de uso, cuáles son los organismos competentes para publicar ciertos datos, además de las políticas definidas para regular y fomentar el uso de la información geográfica.

- Estándares: Los estándares son fundamentales para que una IDE pueda ser interoperable tanto a nivel técnico como a nivel semántico. Se consideran las normas ISO 19100 de Información Geográfica, los estándares OGC y recomendaciones propuestas por las IDEs Nacionales.

- Tecnologías: No es suficiente con tener los datos disponibles a través de internet, es necesario brindar herramientas que permitan buscar, acceder y explorar los datos de manera remota.

- Usuarios: hay distintos tipos de usuarios en función de la finalidad con la que se utilizan los datos. Además aparecen los roles de los generadores de datos y los responsables de su almacenamiento y publicación.

A nivel mudial existe la Global Spatial Data Infrastructure Association $\left(\mathrm{GSDI}^{3}\right)$ que es una organización que promueve el desarrollo de IDEs tanto a niveles regionales como nacionales o locales. En la Unión Europea la Iniciativa INSPIRE ${ }^{4}$ establece las bases para las IDEs de los paises europeos. En particular, la IDE de España (IDEE ${ }^{5}$ ) es un gran ejemplo de IDE de varios niveles. En Estados Unidos existe el Federal Geographic Data Committee $\left(\mathrm{FGDC}^{6}\right.$ ) que es el responsable de la National Spatial Data Infrastructure (NSDI). A nivel regional se destacan la IDE de la República Argentina (IDERA ${ }^{7}$ ) y la IDE de Chile (IDEChile ${ }^{8}$ ).

\footnotetext{
${ }^{3} \mathrm{http}: / /$ gsdiassociation.org

${ }^{4}$ http://inspire.ec.europa.eu/

${ }^{5}$ http://www.idee.es/

${ }^{6}$ https://www.fgdc.gov/

${ }^{7}$ http://www.idera.gob.ar/

${ }^{8}$ http://www.ide.cl/
}

\section{E. Contexto Uruguayo}

Esta sección describe iniciativas en el contexto uruguayo. La Plataforma de Gobierno Electrónico (PGE) del Estado Uruguayo tiene como objetivo general facilitar y promover la implementación de servicios de Gobierno Electrónico en Uruguay [20]. Para esto, la PGE brinda mecanismos que apuntan a simplificar la integración entre los organismos del Estado y a posibilitar un mejor aprovechamiento de sus activos. El soporte tecnológico a la PGE está dado por un conjunto de componentes que implementan la Plataforma de Interoperabilidad y proveen Servicios Transversales. Estos componentes brindan mecanismos para implementar una Arquitectura Orientada a Servicios (Service Oriented Architecture, SOA) a nivel del Estado, garantizar la interacción segura entre los servicios y aplicaciones, e interactuar con los ciudadanos.

En los últimos años, Uruguay se ha sumado a las iniciativas de Datos Abiertos de Gobierno (Open Government Data, OGD) que han surgido en el mundo con el objetivo de lograr que los datos públicos sean de libre acceso para todas las personas [21]. En particular, esta iniciativa ${ }^{9}$ ha disponibilizado un Catálogo de Datos Abiertos ${ }^{10}$ para que la información pública pueda reutilizarse y combinarse con otro contenido digital para crear servicios y productos con un valor agregado. El grado de apertura de los datos puede calificarse utilizando el esquema de cinco estrellas definido por Tim Berners-Lee en el año 2010 [22].

La Ley No 18.331 de Protección de Datos Personales y Acción de "Habeas Data" [23] establece que el derecho a la protección de datos personales es inherente a la persona humana y, por extensión, a las personas jurídicas. La ley especifica un conjunto de datos públicos, que para las personas físicas son nombres y apellidos, documento de identidad, nacionalidad, domicilio y fecha de nacimiento. Además, la ley define datos sensibles como los datos personales que revelan el origen racial, étnico, preferencias políticas, convicciones religiosas o morales, afiliación sindical e informaciones referentes a la salud o a la vida sexual.

\section{F. Infraestructura de Datos Espaciales de Uruguay}

La IDEuy ${ }^{11}$ es la IDE de alcance nacional del Uruguay e involucra a la Administración Central, las Intendencias y varias Empresas Públicas [24]. La IDEUy está organizada como una federación de Nodos Periféricos correspondientes a las Instituciones, donde cada uno publica sus Datos Espaciales y sus Metadatos usando Servicos Web Geográficos. Se considera también un Nodo Central que pueda servir de acceso al público en general para localizar los datos espaciales disponibles. En la Figura 1 se ve la Arquitectura general de la IDEuy.

En el nodo central también es publicada alguna información geográfica espacial, la cual puede ser utilizada desde el GeoPortal de la IDEuy por los diferentes actores como: ciudadanos, investigadores o empresas, entre otros.

\footnotetext{
${ }^{9}$ https://datos.gub.uy/

${ }^{10} \mathrm{https}: / /$ catalogodatos.gub.uy/

${ }^{11} \mathrm{http}: / / w w w . i d e . u y$
} 


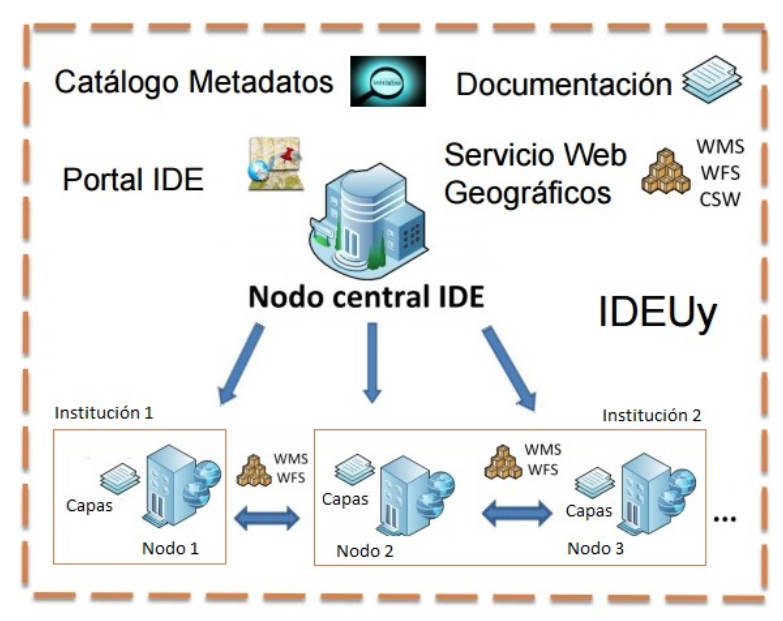

Figura 1: Arquitectura de la IDEuy

A nivel técnico la IDEUy tiene grupos de trabajo que definen normas y la adopción de estándares que aseguren la interoperabilidad de datos y servicios.

\section{IDENTIFICACIÓN DE CARACTERÍ́STICAS DE CALIDAD}

En esta sección se identifican características de calidad para los Servicios Web Geográficos de la IDEuy.

\section{A. Descripción Metodología}

Para identificar características de calidad relevantes para los Servicios Web Geográficos de la IDEuy se analizaron tres tipos de fuentes de información. En primer lugar, se analizaron especificaciones técnicas, requerimientos y recomendaciones del contexto de la IDEuy. En segundo lugar, se analizaron especificaciones técnicas, requerimientos y recomendaciones de otras IDEs de referencia. Por último, se analizaron características identificadas en trabajos relacionados en el área. La Figura 2 presenta gráficamente la metodología seguida.

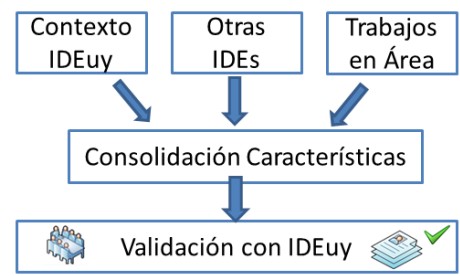

Figura 2: Metodología para Identificación de Características de Calidad

Se llegó entonces a un conjunto consolidado de características de calidad que son la base para el modelo propuesto en la Sección IV. Se validó también con la IDEuy que estas características eran de relevancia para el contexto.

\section{B. Fuentes Relevadas para Identificación de Características}

La identificación de características de calidad del contexto IDEuy se basó en documentación de la IDEuy, documentación de la PGE y entrevistas con referentes de la IDEuy. Algunos documentos técnicos de la IDEuy que se analizaron son los Lineamientos Estratégicos para la Información Geográfica [25], el Modelo de Direcciones Geográficas del Uruguay [26], el Perfil de Metadatos [27], la definición de Sistemas de Referencias y Proyecciones [28]. También se tomaron en cuenta la guía de la PGE y su catálogo de servicios [29].

En cuanto a otras IDEs a nivel internacional, se analizó documentación técnica en lo referente a la calidad de los servicios web en varias IDEs. En particular, se profundizó en el FGDC, en la IDEE, como ejemplo de INSPIRE, y en la IDERA e IDE Chile a nivel regional.

Por último se relevaron propuestas más generales de modelos de calidad de servicios (i.e. OASIS [13], OMG [30], S-Cube [1]) y trabajos académicos en el área de calidad de servicios geográficos y evaluación de calidad en IDEs.

\section{Características de Calidad}

En la Tabla I se presentan las 39 características obtenidas agrupadas en ocho grandes categorías y se referencian en detalle las fuentes a partir de las que fueron identificadas.

La categoría Datos incluye características relativas a la publicación de los datos y al contexto de su publicación. Por ejemplo, Jurisdicción del Dato refiere a que los datos sean del territorio uruguayo y Publicación Instituciones Competentes refiere a que los datos sean publicados por el organismo referente en el tema. Varias características provenientes de otras IDEs refieren a detalles técnicos de la publicación (p. ej. Formato Estilo Capa, Formato PNG, Elementos Gráficos Adicionales, Manejo de Exceso de Rago Útil).

La Categoría Metadatos comprende características relativas a la calidad de los metadatos de los Datos Espaciales y su publicación. Se destacan algunos metadatos significativos como el Plan de Actualización, la Fecha del Dato y la Información de Calidad porque son fundamentales para la IDEuy. Se considera más globalmente la Publicación de los Metadatos y su Completitud. De otras IDEs se toma Leyenda de Capa como un metadato de la publicación de los datos.

La categoría Legislación incluye características identificadas a partir de normativas e iniciativas del contexto uruguayo. En particular, Privacidad surge de lo establecido por la ley 18.331 [23] y Datos Abiertos refiere a los principios de Datos Abiertos de Gobierno en Uruguay.

La categoría Interoperabilidad incluye características que refieren principalmente a cumplir con estándares (p. ej. OGC) o a seguir lineamientos establecidos para el contexto de la IDE (p. ej. Referencia Domiciliaria, Sistema de Referencia). Estas características fueron identificadas principalmente a partir del contexto de la IDEuy y de otras IDEs.

La categoría Seguridad incluye características que refieren a propiedades clásicas de seguridad (p. ej. Autenticación) que fueron identificadas a partir de fuentes del contexto uruguayo y de trabajos en el área de calidad de servicio.

La categoría Operación incluye características relativas al comportamiento en tiempo de ejecución de los servicios (p. ej. disponibilidad, tiempo de respuesta, capacidad). Estas características fueron identificadas principalmente a partir del contexto uruguayo y de trabajos en el área. 
Tabla I: Características de Calidad Identificadas

\begin{tabular}{|c|c|c|c|c|}
\hline Categoría & Característica & Contexto IDEuy & Otras IDEs & Trabajos Académicos en el área \\
\hline \multirow[t]{8}{*}{ Datos } & Jurisdicción del dato & {$[25]$} & & {$[1]$} \\
\hline & Publicación Instituciones Competentes & [25] & & \\
\hline & Correctitud Representación Gráfica & [25] & & \\
\hline & Licencia & & {$[31]$} & \\
\hline & Formato Estilo Capa & & [32] [33] & \\
\hline & Formato PNG & & [32] [34] [33] & \\
\hline & Elementos gráficos adicionales & & {$[32]$} & \\
\hline & Manejo de exceso del rango útil & & [32] [33] & \\
\hline \multirow[t]{7}{*}{ Metadatos } & Plan de Actualización & {$[25][27]$} & & \\
\hline & Fecha del Dato & {$[35]$} & & [1] [36] \\
\hline & Publicación de Metadatos & {$[25]$} & & \\
\hline & Completitud Metadatos & [27] & & [1] \\
\hline & Publicación en Catálogo & [25] & & \\
\hline & Información de Calidad & [25] & & \\
\hline & Leyenda de la Capa & & [32] [33] & \\
\hline \multirow[t]{2}{*}{ Legislación } & Privacidad & {$[23]$} & & \\
\hline & Dato Abierto & {$[25]$} & & \\
\hline \multirow[t]{8}{*}{ Interoperabilidad } & Utilización de Estándar & [25] & [32] & \\
\hline & Sistema de Referencia & {$[25][28]$} & & \\
\hline & Referencia Domiciliaria & {$[26]$} & & \\
\hline & Sistema de Referencia Adicional & & {$[32]$} & \\
\hline & Adopción de Estándares OGC & & [32] [33] [37] & \\
\hline & Formatos de información de capas consultables & & {$[32][33]$} & \\
\hline & Conformidad del estándar & & & {$[13]$} \\
\hline & Múltiples Plataformas & & & [13] \\
\hline \multirow[t]{4}{*}{ Seguridad } & Autenticación & {$[25]$} & & [1] \\
\hline & Integridad & [35] & & \\
\hline & Autorización & [35] & & {$[30][1]$} \\
\hline & Protección & [35] & & {$[38]$} \\
\hline \multirow[t]{7}{*}{ Operación } & Disponibilidad & [29] & & {$[30][39][1]$} \\
\hline & Tiempo de Respuesta & [35] & & {$[40][30]$} \\
\hline & Capacidad & [29] & & {$[40][30]$} \\
\hline & Manejo de Errores & {$[35]$} & & {$[1]$} \\
\hline & Confiabilidad & [35] & & [40] [30] [1] [39] \\
\hline & Formato de Excepción & & [32] [33] & \\
\hline & Accesibilidad & & & {$[13]$} \\
\hline \multirow[t]{2}{*}{ Reputación } & Reputación del servicio & & & {$[13]$} \\
\hline & Reputación Proveedor del servicio & & & [13] [39] \\
\hline Usabilidad & Aprendizaje & & & {$[1][40]$} \\
\hline
\end{tabular}

La categoría Reputación incluye características que hacen referencia a cómo es percibido tanto el proveedor como el servicio por los usuarios del mismo. Estas características se identificaron a partir de trabajo relacionado en el área.

Por último, la categoría Usabilidad hace referencia a la facilidad de uso del servicio (p. ej. documentación que se provee). Esta categoría incluye la característica Aprendizaje que fue identificada a partir de trabajo relacionado en el área.

\section{Propuesta de Modelo de Calidad}

En esta sección se propone un Modelo de Calidad para la evaluación de Servicios Web Geográficos de la IDEuy. En la Sección IV-A se presentan los objetos medibles. En la Sección IV-B se describe la estructura general del modelo en base a dimensiones y factores. Por último, en la Sección IV-C se detallan algunas de las métricas del modelo.

\section{A. Objetos Medibles del Modelo}

Los objetos medibles identificados para el Modelo de Calidad en el contexto de la IDEuy son los siguientes:
- Institución: Representa una entidad proveedora de información geográfica en una IDE. Por ejemplo: la Intendencia de Montevideo (IM) ${ }^{12}$, el Ministerio de Transporte y Obras Públicas (MTOP) ${ }^{13}$, el Ministerio de Vivienda y Ordenamiento Territorial y Medio Ambiente (MVOTMA) ${ }^{14}$.

- Nodo: Representa un servidor que provee servicios web geográficos. Una institución puede tener varios nodos. Por ejemplo, el MVOTMA actualmente tiene dos nodos publicados en la IDEuy ${ }^{15}$.

- Servicio: Representa un servicio web geográfico publicado en un nodo. Por ejemplo, un nodo puede publicar servicios del tipo WMS y WFS.

- Método: Representa un método de un servicio web geográfico. Por ejemplo, el servicio WMS provee los métodos GetCapabilities y GetMap, entre otros.

\footnotetext{
${ }^{12} \mathrm{http}: / /$ sig.montevideo.gub.uy/

${ }^{13} \mathrm{http}: / /$ geoportal.mtop.gub.uy/

${ }^{14} \mathrm{http}: / /$ www.mvotma.gub.uy/

${ }^{15} \mathrm{http}: / /$ ide.uy/nodos-ideuy
} 
- Capa: Representa una capa geográfica publicada en un nodo. Por ejemplo, en el nodo MTOP $^{16}$ se encuentra publicada una capa de rutas nacionales. Una capa puede accederse desde distintos servicios (p. ej. WMS, WFS).

\section{B. Dimensiones y Factores del Modelo}

El modelo propuesto se estructura en dimensiones y factores como se presenta en la Tabla II.

Tabla II: Dimensiones y Factores del modelo propuesto

\begin{tabular}{|l|l|}
\hline Dimensión & Factor \\
\hline Seguridad & Autenticación \\
& Autorización \\
& Integridad \\
& Protección \\
& Privacidad \\
\hline Confiabilidad & Accesibilidad \\
& Disponibilidad \\
& Robustez \\
& Reputación \\
\hline Rendimiento & Tiempo de Respuesta \\
& Capacidad \\
\hline Interoperabilidad & Soporte de Estándares \\
& Sistema de Referencia \\
& Estructura de Datos \\
\hline Publicación Datos & Representación Gráfica \\
& Datos Abiertos \\
& Área de Cobertura \\
& Formatos Soportados \\
& Licencia Datos \\
& Institución Competente \\
\hline Usabilidad & Publicación Catálgo \\
& Metadatos Capa \\
& Metadatos Servicio \\
\hline & Facilidad de Aprendizaje \\
\hline & \\
\hline Metadatos & \\
&
\end{tabular}

La dimensión Seguridad surge directamente de la categoría Seguridad de la Sección III e incluye factores que se corresponden directamente con las características de dicha categoría: Autenticación, Autorización, Integridad y Protección. Además, se incluyó el factor Privacidad que surge de una de las características de la categoría Legislación identificada en la Sección III. Este último factor se incluyó en esta dimensión debido a que se considera un aspecto de la seguridad en varios modelos de QoS (p. ej. en el WSQM).

La dimensión Confiabilidad surge de incluir algunas de las características que se corresponden con la categoría Operación de la Sección III que son: Disponibilidad, Accesibilidad, y Manejo de Errores. Este último se renombró a Robustez, para hacerlo más general como se propone en el modelo S-Cube. Además, se incluyó la categoría Reputación identificada en la Sección III como factor ya que se entendió que es un aspecto de la Confiabilidad del servicio.

La dimensión Rendimiento surge de incluir las características Tiempo de Respuesta y Capacidad de la categoría Operación de la Sección III, dado que se entendió que estas características hacen referencia al rendimiento de los objetos medibles del modelo.

La dimensión Interoperabilidad surge directamente de la categoría Interoperabilidad de la sección III e incluye factores

\footnotetext{
${ }^{16}$ http://geoportal.mtop.gub.uy/geoserv.html
}

que se corresponden con las características de dicha categoría a excepción de Formatos de Información de Capas Consultables que se encuentra en la Dimensión Publicación Datos. Las características que se incluyen en esta dimensión se encuentran agrupadas en tres factores: Soporte de Estándares, Sistema de Referencia y Estructura de Datos. El factor Soporte de Estándares agrupa las características que refieren a estándares (i.e. utilización de estándar, adopción de estándares OGC, múltiples plataformas y conformidad del estándar). El factor Sistema de Referencia agrupa a las características que refieren a sistemas de referencia (i.e. sistema de referencia y sistema de referencia adicional). Por último, el factor Estructura de Datos agrupa a las características que refieren a la estructura de los datos publicados (i.e. referencia domiciliaria).

La dimensión Publicación Datos surge de incluir características de la categoría Datos como el caso de Licencia Datos. Por otro lado, el factor Datos Abiertos surge de la categoría Legislación identificada en la Sección III. Otros factores que se incluyen en la dimensión surgen de la agrupación de características que refieren al mismo aspecto de calidad. Por ejemplo, Representación Gráfica agrupa características que hacen referencia a la representación gráfica de los datos (p. ej. correctitud representación gráfica, elementos gráficos adicionales). De forma similar, el factor Área de Cobertura hace referencia al área geográfica a la que refieren los datos (p. ej. jurisdicción del dato) y el factor Formatos Soportados hace referencia al formato en que se proveen los datos (p. ej. formato PNG). Por último, el factor Institución Competente agrupa características que hacen referencia a la institución dueña del dato (p. ej. publicación instituciones competentes).

La dimensión Metadatos surge directamente de la categoría Metadatos de la sección III. Los factores de esta dimensión se agrupan en tres factores: Publicación Catálogo, Metadatos Capas y Metadatos Servicio. El factor Publicación Catálogo agrupa las características que hacen referencia a la publicación de los metadatos en un catálogo (p. ej. registrado en el catálogo de la IDE, tiene servicio CSW). El factor Metadatos Capa agrupa a las características que hacen referencia a los metadatos de las capas publicadas (p. ej. completitud de metadatos, fecha del dato, plan de actualización, información de calidad). Por último, el factor Metadatos Servicio agrupa características que hacen referencia a los metadatos del servicio (p. ej. leyenda de la capa).

La dimensión Usabilidad surge directamente de la categoría Usabilidad de la sección III y tiene un único factor denominado Facilidad de Aprendizaje, identificado en varios modelos de QoS (p. ej. en el WSQM).

\section{Definición de Métricas}

En esta sección se describen algunas de las métricas del modelo, haciendo foco en las que se considera que están más asociadas a los aspectos geográficos de los servicios. En [35] se pueden consultar todas las métricas del modelo.

Las métricas de los distintos factores surgieron, en algunos casos, directamente de las características que fueron agrupadas en un factor (p. ej. referencia domiciliaria, formato PNG, 
formato de excepción). Otras métricas surgieron de analizar de qué forma se podría medir un determinado factor en los distintos objetos medibles. Cada métrica identificada, se describe en función de los siguientes atributos:

- Nombre: Nombre descriptivo de la métrica.

- Unidad: Unidad en que se mide la métrica.

- Granularidad: Representa el objeto medible al que aplica.

- Semántica: Descripción del procedimiento de medición.

Para la dimensión Seguridad se definieron las métricas que se presentan en la Tabla III.

Tabla III: Métricas de la Dimensión Seguridad

\begin{tabular}{|l|l|}
\hline Factor & Métrica \\
\hline Autenticación & Autenticación de Clientes \\
\hline Autorización & $\begin{array}{l}\text { Autorización a Nivel de Servicio } \\
\text { Autorización a Nivel de Método } \\
\text { Autorización Sobre los Datos }\end{array}$ \\
\hline Integridad & Integridad de Datos \\
\hline Protección & $\begin{array}{l}\text { Expone Métodos Transaccionales } \\
\text { Anti-DoS } \\
\text { Información en Excepciones }\end{array}$ \\
\hline Privacidad & Devuelve Datos Sensibles \\
\hline
\end{tabular}

En particular, la métrica Expone Métodos Transaccionales indica si el servicio expone métodos que permiten modificar datos (p. ej. el método Transaction en un servicio de tipo WFS). La unidad de esta métrica es Boolean y su granularidad es Servicio. La métrica Información en Excepciones indica si las excepciones del servicio se encuentran en algún formato (p. ej. application/vnd.ogc.se_inimage) que evite exponer datos que sean de utilidad para un atacante. La unidad de esta métrica es Boolean y su granularidad es Servicio. La métrica Devuelve Datos Sensibles indica si alguno de los métodos del servicio retorna datos considerados como datos personales sensibles según la Ley $\mathrm{N}^{\circ} 18.331$ de Protección de Datos Personales y acción de "Habeas Data" [23].

Para la dimensión Confiabilidad se definieron las métricas que se presentan en la Tabla IV.

Tabla IV: Métricas de la Dimensión Confiabilidad

\begin{tabular}{|c|c|}
\hline Factor & Métrica \\
\hline Accesibilidad & Accesibilidad Diaria al Nodo \\
\hline Disponibilidad & Disponibilidad del Servicio \\
\hline Robustez & $\begin{array}{l}\text { Errores Diarios del Servicio } \\
\text { Tolerancia a Parámetros Nulos } \\
\text { Tolerancia a Parámetros Largos }\end{array}$ \\
\hline Reputación & $\begin{array}{l}\text { Reputación del Servicio } \\
\text { Reputación del Proveedor } \\
\text { Veracidad de la Capa } \\
\text { Adecuación al Uso }\end{array}$ \\
\hline
\end{tabular}

En particular, la métrica Tolerancia a Parámetros Nulos indica si el método es capaz de retornar errores controlados, cuando es invocado con mensajes con valores nulos en parámetros obligatorios. La unidad de esta métrica es Boolean y su granularidad es Método. La métrica Tolerancia a Parámetros Largos indica si el método es capaz de retornar errores controlados cuando es invocado con mensajes que tienen parámetros que superen el largo permitido. La unidad de esta métrica es Boolean y su granularidad es Método. La métrica
Veracidad de la Capa indica la veracidad de la información de una capa publicada, de acuerdo a la experiencia de los usuarios que la categorizan como Buena (B) o Mala (M). La unidad de esta métrica es una dupla con los porcentajes de valores $\mathrm{M}$ y B con los que fue catogorizada la capa ([M (\%), B (\%)]) y su granularidad es Capa.

Para la dimensión Rendimiento se definieron las métricas que se presentan en la Tabla V.

Tabla V: Métricas de la Dimensión Rendimiento

\begin{tabular}{|l|l|}
\hline Factor & Métrica \\
\hline Tiempo de Respuesta & Promedio Tiempo de Respuesta Diario \\
\hline Capacidad & Máximo de Invocaciones Concurrentes \\
& Tope Máximo de Objetos \\
& Cantidad Invocaciones por Día \\
& Cantidad de Información Diaria \\
\hline
\end{tabular}

En particular, la métrica Tope Máximo de Objetos indica si el nodo especifica un tope máximo de objetos geográficos (p. ej. Rutas) a retornar en un pedido. La unidad de esta métrica es Boolean y su granularidad es Nodo.

Para la dimensión Interoperabilidad se definieron las métricas que se presentan en la Tabla VI.

Tabla VI: Métricas de la Dimensión Interoperabilidad

\begin{tabular}{|l|l|}
\hline Factor & Métrica \\
\hline Soporte de Estándares & Utilización de Estándares OGC \\
& Adopción de Estándares OGC \\
& $\begin{array}{l}\text { Excepciones en Formato OGC } \\
\text { Soporta Múltiples Plataformas } \\
\text { Estilo de Capas en Formato SLD }\end{array}$ \\
\hline Sistema de Referencia & $\begin{array}{l}\text { Capas del Servicio con CRS Adecuado } \\
\text { (IDEuy) } \\
\text { Capa con CRS Adecuado (IDEuy) } \\
\text { Transformación de CRS }\end{array}$ \\
\hline Estructura de Datos & Referencia Domiciliaria \\
\hline
\end{tabular}

En particular, la métrica Adopción de Estándares OGC representa el nivel de implementación del estándar OGC en los métodos del servicio web geográfico, verificando si implementa los métodos básicos o también los opcionales que propone el estándar. Esta métrica puede tomar tres valores posibles (i.e. Básico, Intermedio, Completo) y su granularidad es Servicio. La métrica Estilo de Capas en Formato SLD indica si el servicio utiliza la especificación OGC "Styled Layer Descriptor Implementation Specification"17 para simbolización de las capas. La unidad de esta métrica es Boolean y su granularidad es Servicio. La métrica Capas del Servicio con CRS Adecuado indica el porcentaje del total de las capas publicadas por el servicio que utilizan el CRS establecido por la IDE Nacional. La unidad de esta métrica es Porcentaje y su granularidad es Servicio. La métrica Capa con CRS Adecuado indica si la capa que es publicada por el servicio cumple con el CRS establecido por la IDE Nacional. La unidad de esta métrica es Boolean y su granularidad es Capa.

Para la dimensión Publicación Datos se definieron las métricas que se presentan en la Tabla VII.

\footnotetext{
${ }^{17}$ http://www.opengeospatial.org/standards/sld
} 
Tabla VII: Métricas de la Dimensión Publicación Datos

\begin{tabular}{|l|l|}
\hline Factor & Métrica \\
\hline Representación Gráfica & $\begin{array}{l}\text { Soporta Imagen Simplificada } \\
\text { Soporta Imagen Vacía } \\
\text { Elementos Gráficos Adicionales } \\
\text { Correctitud Representación Gráfica }\end{array}$ \\
\hline Datos Abiertos & Grado de Apertura de los Datos \\
\hline Área de Cobertura & Jurisdicción del Dato \\
\hline Formatos Soportados & Formato PNG \\
& Formato KML \\
& Formato text/html Método GetFeatureInfo \\
& Formato text/xml Método GetFeatureInfo \\
& Formato Excepción vnd.ogc.se_inimage \\
& Formato Excepción vnd.ogc.se_blank \\
& Cantidad de Formatos Soportados \\
& Cantidad de Formatos Excepciones Soportadas \\
\hline Licencia Datos & Licenciamiento Creative Commons \\
\hline Institución Competente & Publicación Datos Competentes \\
\hline
\end{tabular}

En particular, la métrica Correctitud Representación Gráfica indica el porcentaje de las capas del nodo que cumplen con la temática definida ( $p$. ej. controlar los colores utilizados en las capas, según los objetos geográficos establecidos). La unidad de esta métrica es el porcentaje del total de capas que cumplen con la representación gráfica y su granularidad es Nodo. La métrica Grado de Apertura de los Datos indica el grado de apertura de los datos geográficos que son publicados. La unidad de esta métrica es la clasificación de estrellas ${ }^{18}$ y su granularidad es Capa. La métrica Jurisdicción del Dato indica el porcentaje de capas que referencian zonas geográficas donde se tiene soberanía y jurisdicción. La unidad de la métrica es porcentaje total de las capas del nodo y su granularidad es Nodo. La métrica Formato PNG indica si el método getMap() del servicio WMS soporta el formato Portable Network Graphics (image/png). La unidad de la métrica es Boolean y su granularidad es Método. La métrica Formato text/html indica si el método getFeatureInfo() del servicio WMS soporta el formato text/html. La unidad de la métrica es Boolean y su granularidad es Método. La métrica Publicación Datos Competentes indica cuántas capas que son publicadas por la institución, son de su competencia. La unidad de la métrica es el porcentaje de capas que son de su competencia y su granularidad es Institución.

Para la dimensión Metadatos se definieron las métricas que se presentan en la Tabla VIII.

Tabla VIII: Métricas de la Dimensión Metadatos

\begin{tabular}{|l|l|}
\hline Factor & Métrica \\
\hline Publicación Catálogo & $\begin{array}{l}\text { Registrado en el Catálogo Metadatos IDE } \\
\text { Tiene Servicio CSW (Servicio Web de Catálogo) }\end{array}$ \\
\hline Metadatos Capa & $\begin{array}{l}\text { Tiene Metadatos } \\
\text { Publicación de Acuerdo al Perfil } \\
\text { Información de Calidad }\end{array}$ \\
& Fecha del Dato \\
& Completitud de Metadatos \\
& Plan de Actualización \\
\hline Metadatos Servicio & Leyenda de la Capa \\
& Especifica Rango Útil \\
\hline
\end{tabular}

\footnotetext{
${ }^{18} \mathrm{http}: / / 5$ stardata.info/en/
}

En particular, la métrica Tiene Servicio CSW indica si la institución que publica los datos geográficos, proporciona un servicio del tipo CSW para la búsqueda de los metadatos. La unidad de esta métrica es Boolean y su granularidad es Institución. La métrica Tiene Metadatos indica el porcentaje de los datos geográficos que son publicados y tienen sus respectivos metadatos. La unidad de esta métrica es un Porcentaje y su granularidad es Institución. La métrica Publicación de Acuerdo al Perfil indica el porcentaje de los metadatos que son publicados de acuerdo al perfil para los metadatos. La unidad de esta métrica es un Porcentaje y su granularidad es Institución. La métrica Información de Calidad indica el porcentaje de los metadatos publicados que tienen información sobre la calidad de dichos datos. La unidad de esta métrica es un Porcentaje y su granularidad es Institución. La métrica Fecha del Dato indica el porcentaje de los metadatos que tienen en sus datos la fecha en la cual se generó el dato. La unidad de esta métrica es un Porcentaje y su granularidad es Institución. La métrica Plan de Actualización indica si los datos publicados tienen en sus metadatos un plan de actualización público y de fácil acceso. La unidad de esta métrica es Boolean y su granularidad es Capa, La métrica Leyenda de la Capa indica si el servicio WMS implementa el método GetLegendGraphic. La unidad de esta métrica es Boolean y su granularidad es Servicio.

Para la dimensión Usabilidad se definieron las métricas que se presentan en la Tabla IX.

Tabla IX: Métricas de la Dimensión Usabilidad

\begin{tabular}{|l|l|}
\hline Factor & Métrica \\
\hline Facilidad de Aprendizaje & Errores Descriptivos \\
& Ejemplos \\
& Documentación \\
\hline
\end{tabular}

La métrica Documentación indica si la institución brinda algún tipo documentación de uso de sus servicios (p. ej. ejemplos de invocación). La unidad de esta métrica es Boolean y su granularidad es Institución.

\section{Aplicación del Modelo}

En esta sección se describe la aplicación del modelo propuesto para evaluar cinco servicios geográficos de la IDEuy.

\section{A. Definición de Perfil de Evaluación}

Para evaluar la calidad de los servicios web geográficos se propone la definición de perfiles. Un perfil de evaluación es un conjunto de métricas aplicables a un mismo objeto medible (p. ej. servicio) y rangos de valores esperados para las mismas según el objetivo de la evaluación. La Tabla X presenta un perfil de evaluación básico, aplicable al objeto servicio, que incluye métricas consideradas de interés para la IDEuy.

\section{B. Evaluación de Servicios Web Geográficos de la IDEuy}

Para la evaluación de los Servicio Web geográficos de la IDEuy se consideraron los siguientes nodos: Ministerio de Transporte y Obras Públicas (MTOP) ${ }^{19}$, el Servicio Geográfico

\footnotetext{
${ }^{19}$ http://geoportal.mtop.gub.uy/geoserv.html
} 
Tabla X: Métricas del Perfil de Evaluación Básico

\begin{tabular}{|l|l|l|}
\hline Métrica & Valor Esperado & Dimensión \\
\hline Disponibilidad del Servicio & $>70 \%$ & Confiabilidad \\
\hline $\begin{array}{l}\text { Capas del Servicio con CRS } \\
\text { Adecuado (IDEuy) }\end{array}$ & $>=50 \%$ & Interoperabilidad \\
\hline Estilo Capas en Formato SLD & $\mathrm{Si}$ & Interoperabilidad \\
\hline $\begin{array}{l}\text { Cantidad de Formatos de Ex- } \\
\text { cepciones Soportadas }\end{array}$ & $>=2$ & $\begin{array}{l}\text { Publicación Da- } \\
\text { tos }\end{array}$ \\
\hline
\end{tabular}

Militar (SGM) $)^{20}$ y la Intendencia de Montevideo (IM) ${ }^{21}$. En la tabla XI se presentan los servicios evaluados.

Tabla XI: Servicios Considerados en la Evaluación

\begin{tabular}{|l|l|l|}
\hline Nodo & ID Servicio & Descripción \\
\hline MTOP & MTOP_1 & WMS Logística \\
& MTOP_2 & WMS Recursos Hidrográficos \\
\hline SMG & SGM_1 & WMS Ciudad Artigas \\
& SGM_2 & WMS Centros Poblados Artigas \\
\hline SMG & IM_1 & WSM Intendencia de Montevideo \\
\hline
\end{tabular}

La Tabla XII presenta los resultados de la medición y si el servicio cumple (C) o no cumple (NC) con el requerimiento.

Tabla XII: Resultado Evaluación del Perfil Básico

\begin{tabular}{|c|c|c|c|c|c|}
\hline & $\begin{array}{l}\vec{b} \\
\bar{o} \\
\underline{z}\end{array}$ & 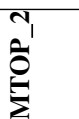 & $\sum_{0}^{\prime}$ & 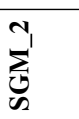 & $Z^{\prime}$ \\
\hline $\begin{array}{l}\text { Disponibilidad del servi- } \\
\text { cio }\end{array}$ & $\begin{array}{l}100 \% \\
(C)\end{array}$ & $\begin{array}{l}100 \% \\
(C)\end{array}$ & $\begin{array}{l}100 \% \\
(C)\end{array}$ & $\begin{array}{l}100 \% \\
(C)\end{array}$ & $\begin{array}{l}100 \% \\
(C)\end{array}$ \\
\hline $\begin{array}{l}\text { Capas del Servicio con } \\
\text { CRS Adecuado (IDEuy) }\end{array}$ & $\begin{array}{l}0 \% \\
(N C)\end{array}$ & $\begin{array}{l}0 \% \\
(N C)\end{array}$ & $\begin{array}{l}0 \% \\
(N C)\end{array}$ & $\begin{array}{l}0 \% \\
(N C)\end{array}$ & $\begin{array}{l}0 \% \\
(N C)\end{array}$ \\
\hline $\begin{array}{l}\text { Estilo de Capas en Forma- } \\
\text { to SLD }\end{array}$ & $\begin{array}{l}\mathrm{Si} \\
(C)\end{array}$ & $\begin{array}{l}\mathrm{Si} \\
(C)\end{array}$ & $\begin{array}{l}\text { No } \\
(N C)\end{array}$ & $\begin{array}{l}\text { No } \\
(N C)\end{array}$ & $\begin{array}{l}\mathrm{Si} \\
(C)\end{array}$ \\
\hline $\begin{array}{l}\text { Cantidad de formatos de } \\
\text { excepciones soportadas }\end{array}$ & $2(C)$ & $2(C)$ & $3(C)$ & $3(C)$ & $3(C)$ \\
\hline
\end{tabular}

Los servicios del MTOP e IM cumplen entonces con un $75 \%$ de los requerimientos de calidad, mientras que los servicios del SGM cumplen un $50 \%$ de los requerimientos.

\section{Detalles de Implementación}

Para evaluar los servicios web geográficos se implementó un prototipo en Java que implementa métodos para algunas de las métricas del modelo. Por ejemplo, la implementación de un método de la métrica Cantidad de Formatos de Excepciones Soportadas invoca el método GetCapabilities y obtiene la cantidad de formatos soportados del XML que retorna dicho método, como se presenta en la Figura 3.

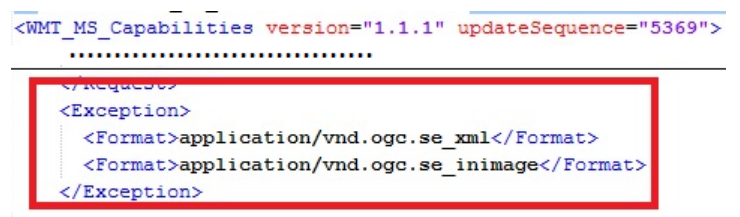

Figura 3: GetCapabilities Formato de Excepciones

\footnotetext{
${ }^{20} \mathrm{http}: / /$ www.sgm.gub.uy/geoportal/index.php/geoservicios/listado-deservicios

${ }^{21} \mathrm{http}: / /$ sig.montevideo.gub.uy/content/geoservicios-web
}

\section{TRABajo RElacionado}

Se han elaborado varios modelos de calidad para servicios web dentro de los que se destacan el Web Services Quality Model de OASIS [13], el perfil para modelar calidad de servicio de la OMG [30] y el modelo desarrollado en el proyecto S-Cube [1]. Si bien varias características de estos modelos fueron aprovechadas en este trabajo, nuestra propuesta además contempla aspectos específicos de los servicios web geográficos (p. ej. representación gráfica, publicación de metadatos, sistema de referencia).

Por otro lado, existen también trabajos en el área de calidad de servicios web geográficos [40][39][41][5]. Sin embargo, estos no contemplan algunos lineamientos requeridos por la IDEuy como es la jurisdicción del dato, sistema de referencia, publicación metadatos, entre otros. Además dichos trabajos no proponen un modelo de calidad, sino que presentan características de forma independiente.

Por último, existen trabajos en el área de calidad de servicio en el contexto de una IDE [7][36][38]. A diferencia de nuestro trabajo, estos no proponen un modelo de calidad y no contemplan algunos lineamientos de la IDEuy.

\section{ViI. Conclusiones y Trabajo a Futuro}

En este artículo se propone un Modelo de Calidad para la Evaluación de Servicios Web Geográficos en la IDEuy. El mismo se construyó en base a características de calidad identificadas en requerimientos del contexto uruguayo (p. ej. en la IDEuy), IDEs de referencia y trabajo relacionado en el área. El modelo propuesto consta de 7 dimensiones, 24 factores y 61 métricas, las cuales pueden utilizarse para medir los distintos objetos contemplados por el modelo: institución, nodo, servicio, método y capa. El trabajo también propone la definición de perfiles para realizar la evaluación de la calidad de dichos objetos y presenta un prototipo que implementa métodos de medición para algunas de las métricas.

Los principales aportes del trabajo son el análisis y consolidación de características de calidad para servicios web geográficos de la IDEuy, la elaboración de un modelo de calidad extensible en base a las mismas, la posibilidad de definir perfiles para la evaluación de la calidad y el desarrollo de un prototipo para evaluar servicios de la IDEuy, comprobando la factibilidad técnica de la propuesta. Entendemos que estos aportes constituyen un paso importante para promover la calidad de los servicios web geográficos en la IDEuy.

Actualmente, se está trabajado en la construcción de una herramienta web que permita gestionar los distintos objetos de una IDE, gestionar modelos de calidad de servicio, definir perfiles de evaluación, realizar evaluaciones de servicios web geográficos y generar reportes en base a las evaluaciones.

A futuro se planea definir perfiles de evaluación más avanzados de forma de brindar una hoja de ruta a las instituciones hacia mayores niveles de calidad para los servicios web geográficos que proveen.

Por último, si bien la propuesta se enmarcó en el contexto de la IDEuy, entendemos que es también aplicable a otras IDEs con características similares. 


\section{AGRADECIMIENTOS}

Este trabajo fue parcialmente financiado por CSIC, Universidad de la República, Uruguay.

\section{REFERENCIAS}

[1] C. Cappiello, K. Kritikos, A. Metzger, M. Parkin, B. Pernici, P. Plebani, and M. Treiber, "A quality model for service monitoring and adaptation," Madrid, Spain, Jun. 2009, pp. 29-42.

[2] Y. Kang, "Extended Model Design for Quality Factor Based Web Service Management," in Proceedings of the Future Generation Communication and Networking - Volume 02, ser. FGCN '07. Washington, DC, USA: IEEE Computer Society, 2007, pp. 484-487.

[3] D. A. Menascé, "QoS Issues in Web Services," IEEE Internet Comput., vol. 6, pp. 72-75, Nov. 2002.

[4] A. F. R. P. Paloma Abad Power, Miguel A. Bernabé, "Compartir: La solución está en las infraestructuras de datos espaciales," in Fundamentos de las Infraestructuras de Datos Espaciales, C. M. Bernabé Poveda, Miguel Ángel; López Vázquez, Ed. UPM Press, 2012.

[5] P. Medeiros, "Quality assessment for geographic web services," 2009.

[6] G. Subbiah, A. Alam, L. Khan, and B. Thuraisingham, "Geospatial data qualities as web services performance metrics," in Proceedings of the 15th annual ACM international symposium on Advances in geographic information systems. ACM, 2007, p. 66.

[7] I. Simonis and A. Sliwinski, "Quality of service in a global SDI." in From Pharaohs to Geoinformatics, FIG Working Week 2005 and GSDI8.

[8] E. F. d. C. Juan M. Hernández Faccio, "Características de la información geográfica," in Fundamentos de las Infraestructuras de Datos Espaciales, C. M. Bernabé Poveda, Miguel Ángel; López Vázquez, Ed. UPM Press, 2012.

[9] F. J. E. Javier Moya Honduvilla, Miguel A. Bernabé, "La representación de la información geográfica," in Fundamentos de las Infraestructuras de Datos Espaciales, C. M. Bernabé Poveda, Miguel Ángel; López Vázquez, Ed. UPM Press, 2012.

[10] OGC®) Geography Markup Language (GML), OGC Std. 3.3, 2012. [Online]. Available: http://www.opengeospatial.org/standards/gml

[11] $O G C$ KML 2.3, OGC Std. 2.3, 2015. [Online]. Available: http://www.opengeospatial.org/standards/kml

[12] S. Ran, "A model for web services discovery with QoS," ACM SIGecom Exchanges, vol. 4, pp. 1-10, Mar. 2003.

[13] E. Kim, Y. Lee, Y. Kim, H. Park, J. Kim, B. Moon, J. Yun, and G. Kang, Web Services Quality Factors, OASIS Std., Jul. 2011. [Online]. Available: http://docs.oasis-open.org/wsqm/WS-QualityFactors/v1.0/cs01/WS-Quality-Factors-v1.0-cs01.html

[14] L. Etcheverry, V. Peralta, and M. Bouzeghoub, "Qbox-Foundation: a Metadata Platform for Quality Measurement," in "Proceedings of the 4eme Atelier Qualité des données et des Connaisances”, Nice, France, 2008.

[15] OpenGISR Web Map Server Implementation Specification, OGC Std. 1.3.0, 2006. [Online]. Available: http://www.opengeospatial.org/standards/wms

[16] OGC® Web Feature Service 2.0 Interface Standard - With Corrigendum, OGC Std. 2.0.2, 2014. [Online]. Available: http://www.opengeospatial.org/standards/wfs

[17] OGC® WCS 2.0 Interface Standard-Core: Corrigendum, OGC Std. 2.0, 2012. [Online]. Available: http://www.opengeospatial.org/standards/wcs

[18] OGC® Catalogue Services 3.0 Specification, OGC Std. 3.0, 2016 [Online]. Available: http://www.opengeospatial.org/standards/cat

[19] C. Bernabé-Poveda, M.A.;López-Vázquez, Fundamentos de las infraes tructuras de datos espaciales. Madrid: UPM Press, 2012.

[20] L. González, R. Ruggia, J. Abin, G. Llambías, R. Sosa, B. Rienzi, D. Bello, and F. Alvarez, "A Service-oriented Integration Platform to Support a Joined-up E-government Approach: The Uruguayan Experience," in Joint International Conference on Electronic Government, the Information Systems Perspective, and Electronic Democracy, Austria, Sep. 2012.

[21] E. Kalampokis, E. Tambouris, and K. Tarabanis, Electronic Government: 10th IFIP WG 8.5 International Conference, EGOV 2011, Delft, The Netherlands, August 28 - September 2, 2011. Proceedings. Berlin, Heidelberg: Springer Berlin Heidelberg, 2011, ch. Open Government Data: A Stage Model, pp. 235-246.
[22] T. Heath and C. Bizer, Linked Data: Evolving the Web into a Global Data Space, 1st ed. Morgan \& Claypool, 2011. [Online]. Available: http://linkeddatabook.com/

[23] Parlamento Uruguayo, "Ley no 18.331. protección de datos personales y acción de "habeas data"," 2008, http://www.impo.com.uy/bases/leyes/18331-2008.

[24] SIDE, "Infraestructura de datos espaciales para uruguay informe de subgrupo infraestructura de datos espaciales (IDE)," Tech. Rep., 2006.

[25] AGESIC, "Lineamientos Estratégicos para la Información Geográfica de la IDEuy," Tech. Rep., 2013.

[26] AGESIC , "Modelo de direcciones geograficas del uruguay," AGESIC, Tech. Rep., 2012.

[27] AGESIC, "Información Geográfica - Perfil de Metadatos," Tech. Rep., 2013.

[28] AGESIC , "Sistemas de Referencias / Sistemas de Proyecciones Infraestructura de Datos Espaciales del Uruguay." Tech. Rep., 2013.

[29] AGESIC, "Guía de uso de la Plataforma de GE del Estado uruguayo," Tech. Rep., 2011.

[30] Object Management Group (OMG), UML Profile for Modeling Quality of Service and Fault Tolerance Characteristics and Mechanisms Specification, Std., Apr. 2008.

[31] IDERA, "Lineamientos para el acceso, difusión, uso e interoperabilidad de información geoespacial," IDERA, Tech. Rep., 2015.

[32] IDERA, "Reomendaciónes para Servicios Web de Mapas WMS," Tech. Rep., 2014.

[33] INSPIRE, "Technical guidance view services v3.1," INSPIRE, Tech. Rep., 2013.

[34] I. Chile, "Recomendaciones para crear y configurar web map service(wms)," IDE Chile, Tech. Rep., 2012.

[35] Martín de los Reyes, "Modelo de Calidad para la Evaluación de Servicios Web Geográficos en la Infraestructura de Datos Espaciales de Uruguay," Tesis de Maestría en Sistemas de Información y Tecnologías de Gestión de Datos, Centro de Posgrados y Actualización Profesional en Informática - Instituto de Computación - Facultad de Ingeniería Universidad de la República, Dec. 2016.

[36] S. Gao, D. Mioc, and X. Yi, "The measurement of geospatial web service quality in SDIs," in 2009 17th International Conference on Geoinformatics, Aug 2009, pp. 1-6.

[37] INSPIRE, "Technical guidance discovery services v3.1," INSPIRE, Tech. Rep., 2011.

[38] S. Gao, D. Coleman, C. MacLachlan, and G. Plunkett, "CGDI in action: Exploring quality of service,” ISPRS Archives, vol. 38, no. 1, p. 79, 2010.

[39] P. Yue, Z. Tan, and M. Zhang, "GeoQoS: delivering quality of services on the geoprocessing web," in Proceedings of OSGeo European Conference on Free and Open Source Software for Geospatial, 2014, pp. $15-17$.

[40] H. Wu, Z. Li, H. Zhang, C. Yang, and S. Shen, "Monitoring and evaluating the quality of web map service resources for optimizing map composition over the internet to support decision making," Computers and Geosciences, vol. 37, no. 4, pp. 485 - 494, 2011.

[41] L. Miao, X. Shi, and S. Cao, "A scoring model for evaluating geospatial web services: A case study of OGC Web Map Service, year=2011, pages $=1-4$, issn=2161-024X, month=June," in 2011 19th International Conference on Geoinformatics. 